Research Paper

\title{
Proton-sensing GPCR-YAP Signalling Promotes Cancer- associated Fibroblast Activation of Mesenchymal Stem Cells
}

\author{
Hongyi Zhu' ${ }^{*}$, Shangchun Guo ${ }^{2 *}$, Yuelei Zhang ${ }^{1}$, Junhui Yin², Wenjing Yin ${ }^{1}$, Shicong Tao ${ }^{1}$, Yang Wang ${ }^{2}$, \\ Changqing Zhang $1,2 \bowtie$ \\ 1. Department of Orthopaedic Surgery, Shanghai Jiaotong University Affiliated Sixth People's Hospital, Shanghai 200233, China; \\ 2. Institute of Microsurgery on Extremities, Shanghai Jiaotong University Affiliated Sixth People's Hospital, Shanghai 200233, China. \\ "These authors contribute equally to this work. \\ $\triangle$ Corresponding author: Changqing Zhang; Tel: +86 21 64369181; Fax: +86 216470 1361; Email: zhangcq@sjtu.edu.cn.
}

() Ivyspring International Publisher. Reproduction is permitted for personal, noncommercial use, provided that the article is in whole, unmodified, and properly cited. See http://ivyspring.com/terms for terms and conditions.

Received: 2015.08.29; Accepted: 2015.12.22; Published: 2016.02.05

\begin{abstract}
The $\mathrm{pHs}$ of extracellular fluids (ECFs) in normal tissues are commonly maintained at 7.35 to 7.45 . The acidification of the ECF is one of the major characteristics of tumour microenvironment. In this study, we report that decreased extracellular $\mathrm{pH}$ promotes the transformation of mesenchymal stem cells (MSCs) into cancer-associated fibroblasts (CAFs), termed CAF activation. Furthermore, we demonstrate that GPR68, a proton-sensing G-protein-coupled receptor (GPCR), is required for the $\mathrm{pH}$-dependent regulation of the differentiation of MSCs into CAFs. We then identify Yes-associated protein 1 (YAP) as a downstream effector of GPR68 for CAF activation. Finally, we show that knockdown of GPR68 in MSCs can prevent the CAF activation under cancer microenvironment. Systemic transplantation of GPR68-silenced MSCs suppresses in-situ tumour growth and prolong life span after cancer graft.
\end{abstract}

Key words: pH, YAP, proton-sensing, GPCR, cancer, fibroblast.

\section{Introduction}

Cancer-associated fibroblasts represent one of the most abundant stromal cell types that surround cancer cells. CAFs are activated fibroblasts that share similar phenotypes with fibroblasts stimulated by inflammatory or wound healing conditions [1, 2]. Multiple molecular markers of CAF have been well-established including a-smooth muscle actin (aSMA), vimentin, tenascin $\mathrm{C}$, fibroblast-specific protein 1 (FSP1) and neuron glial antigen 2 (NG2) [3, 4]. CAFs influence tumour growth, angiogenesis, metastasis, immunogenicity, and resistance to therapies by producing several secreted factors such as interleukin 6 (IL-6), CXCL8, CXCL12, and epidermal growth factor (EGF) [5-7].

Although naive MSCs can inhibit tumour growth by secretion of multiple tumour-suppressing factor such as DKK1 and oncostatin M [8, 9], MSCs can also differentiate into CAFs which promote tumour growth $[3,10,11]$. Several characteristics of tumour microenvironment including enriched transforming growth factor $\beta$ (TGF $\beta$ ) and stiff extracellular matrix have been shown associated with the CAF activation of MSCs $[12,13]$.

Decrease in extracellular $\mathrm{pH}$ is a major feature of cancer-associated microenvironment. Cancer cells commonly catabolize glucose to lactate for energy generation even when oxygen is plentiful, which is termed the Warburg Effect. In addition, rapid proliferation of cancer cells together with limited angiogenesis leads to hypoxia in tumours. Hypoxia and the Warburg effect lead to the acidification of the extracellular environment in tumours. We therefore hypothesized that low extracellular $\mathrm{pH}$ might be involved in CAF activation of MSCs. 
In this study, we first demonstrate decreased ECF $\mathrm{pH}$ promotes CAF activation of MSCs through GPR68, a proton-sensing GPCR. We then show YAP downstream of GPR68 is required for $\mathrm{pH}$-dependent CAF activation. Finally, we show that knockdown of GPR68 in MSCs can prevent the CAF activation under cancer microenvironment. Mice receiving systemic transplantation of GPR68-silenced MSCs showed suppressed in-situ tumour growth and prolonged life span after cancer graft.

\section{Materials and methods}

\section{Study approval}

The animal study was approved by the IACUC of Shanghai Jiaotong University School of Medicine. The use of human MSCs in this study was approved by the ethics committee of Shanghai Jiaotong University Affiliated Sixth People's Hospital. Informed consents were obtained from all donors in accordance with the Declaration of Helsinki.

\section{Cell isolation and culture}

The MSCs were isolated from cancellous bones of healthy donors (age 30-50 years old) obtained during the orthopaedic operations. Briefly, the cancellous bone was flushed with culture medium and the medium was then transferred to a T75 container for adherence isolation of MSCs. After passage to P2, the MSC surface markers were routinely examined by flow cytometry (Guava) and summarized in Supplementary Table 1 . The MSCs were cultured in high-glucose DMEM (Invitrogen) with 10\% FBS (Gibco) on matrigel-coated plates or dishes. The human breast cancer cells (MDA-MB-453) and gastric carcinoma cells (MKN1) were cultured with RPMI-1640 (Corning) with 5\% FBS. The human ovarian cancer cells (SKOV-3) were cultured with DMEM/F12 (Corning) with 10\% FBS. For clone separation, we plated $50 \sim 100$ MSCs per $10 \mathrm{~cm}$ dish. Colonies were picked up by cloning cylinder after reaching more than 200 cells. The MSC surface markers along with GPR68 were routinely detected by flow cytometry. The clones with highest and lowest GPR68 expression in 20 clones were defined as GPR68-high and GPR68-low clones respectively.

Different $\mathrm{pH}$ values of media were achieved by adding $1 \mathrm{M} \mathrm{HCl}$ or $1 \mathrm{M} \mathrm{NaOH}$ to the medium after $\mathrm{pH}$ equilibrium in the incubator for 24 hours. The media were replaced every $12 \mathrm{~h}$ to ensure the stability of the extracellular pHs in this study.

\section{RNA Extraction, Reverse Transcription and Real-Time PCR}

We used Trizol (Invitrogen) reagents to isolate RNA from samples according to the manufacturer's instructions. Before reverse transcription, the RNA quality and quantity were detected with a NanoDrop 2000. Reverse transcription was then conducted using the Transcript First Strand cDNA Synthesis Kit (Transgene). Real-time PCR was finally performed using FS Universal SYBR Green Master (Roche) on the ABI 7900HT (Applied Biosystems). Each experiment was performed at least three times. GAPDH was adopted as the internal control for the normalisation. The detailed information about the primers is shown as follows:

hIL6-FOR: ACCCCCAATAAATATAGGACTGGA

hIL6-REV: GAAGGCGCTTGTGGAGAAGG

hCXCL8-FOR: GATGGGTGGAGTCGCGTG

hCXCL8-REV: ACCGGAAGGAACCATCTCAC

hCXCL12-FOR: GCCCTTCAGATTGTAGCCCG

hCXCL12-REV: GCGTCTGACCCTCTCACATC

hEGF-FOR: TCCAAGTGCATCAACACCGA

hEGF-REV: GGGTGGAGTAGAGTCAAGACA

hCSPG4-FOR: CTGGTCCGGCACAAGAAGAT

hCSPG4-REV: ATACGATGTCTGCAGGTGGC

Other primer sequences were described previously [14].

\section{Lentivirus infection and RNA interference}

All lentiviruses used in this study were purchased from Genepharm (China). For lentivirus infection, the cells were commonly incubated in medium with lentiviruses and $5 \mu \mathrm{g} / \mathrm{mL}$ polybrene for twenty-four hours. The infected cells were then selected with $2.5 \mu \mathrm{g} / \mathrm{mL}$ puromycin (Sigma-Aldrich) in culture medium for future experiments forty-eight hours after infection. The sequences for RNA interference were described previously [14].

\section{Flow cytometry detection}

Cultured cells were first dissociated with trypsin (Corning) from plates into single-cell suspension. For intracellular marker detection, cells were fixed, permeabilized and blocked. The cells were then incubated with primary antibody for one hour. For surface marker detection, cells were blocked and then incubated with primary antibodies for one hour. After primary antibody incubation, Alexa-conjugated secondary antibodies (Cell Signaling Technologies) were then added to cell suspension for thirty minutes before FACS detection. The GPR68 antibody (sc-98437) was obtained from Santa Cruz. The aSMA antibody (ab119952) was obtained from Abcam. For analysis of subpopulation in tumours, cells were first dissociated from tumours using $0.25 \%$ trypsin for $30 \mathrm{~min}$ and $0.1 \%$ collagenase for 24 hours into single cell suspension.

\section{Animals}

Six-weeks-old female nude mice were maintained in Institute of Animal Experiment, Shanghai 
Jiaotong University Affiliated Sixth People's Hospital. SKOV-3 $\left(5 \times 10^{6}\right)$ and MDA-MB-453 $\left(5 \times 10^{6}\right)$ cells with or without MSCs $\left(5 \times 10^{6}\right)$ suspended in equal volumes of $50 \%$ Matrigel (diluted with DMEM, Corning) were injected subcutaneously into the nude mice. For systemic transplantation of MSCs, $5 \times 10^{5}$ MSCs were suspended in $0.1 \mathrm{~mL}$ saline and then injected through caudal veins every three days after transplantation of tumour cell subcutaneously. The tumour volumes were calculated according to the following: volume $=0.5 \times(\text { width })^{2} \times$ length one month after graft.

\section{Statistics}

Statistical analyses were performed using OriginPro 2015 (OriginLab). Numerical values are represented as the mean \pm SD of at least three independent experiments. A P-value of less than 0.05 was considered statistically significant when an unpaired, two-tailed Student's $t$ test or one-way ANOVA was used.
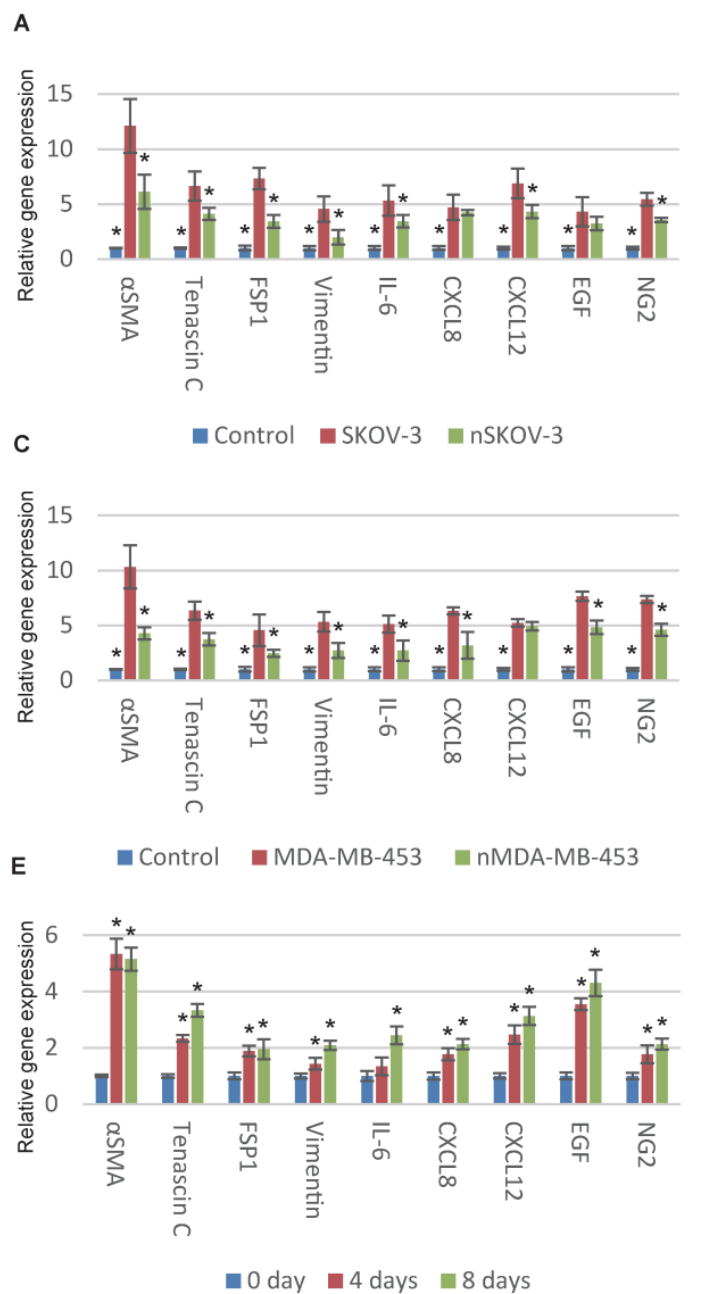

\section{Results}

\section{Decreased ECF pH promotes CAF activation}

We began with examining the relationship between ECF $\mathrm{pH}$ and CAF activation of MSCs. We first normalized the $\mathrm{pH}$ of cancer-cell-cultured medium from approximately 7.0 to 7.4 and then stimulated MSCs with these medium. Compared with original medium, the CAF-specific genes were significantly downregulated after $\mathrm{pH}$ normalization indicating the decreased $\mathrm{pH}$ is important for cancer cells to transform MSCs to CAFs (Fig. 1A, 1B and 1C). We then cultured MSCs with normal medium at different $\mathrm{pHs}$. Consistent with previous observation, decreased ECF $\mathrm{pH}$ induces the upregulation of CAF-specific genes (Fig. 1D and S1A). The time-dependent expression of CAF-specific genes in MSCs was also observed (Fig. $1 \mathrm{E}$ and $\mathrm{S1B})$. CAF activation was partially reversed after culturing in normal pH conditions (Fig. S1C).
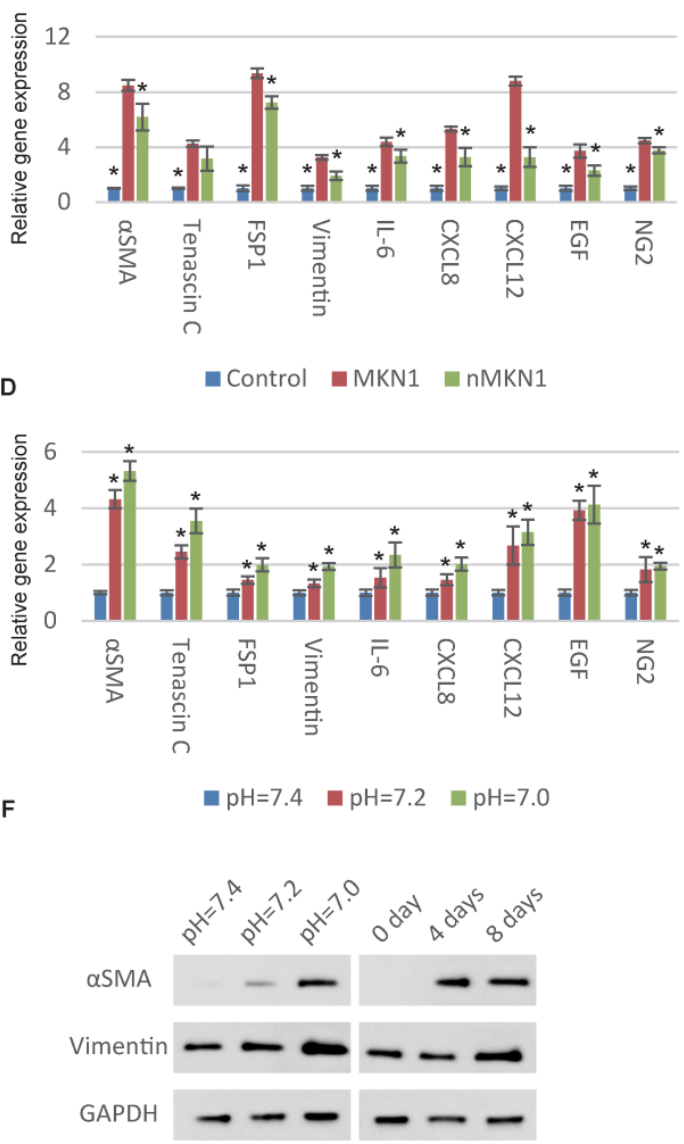

Figure 1. Decrease in $\mathrm{pH}$ induces CAF activation of MSCs. (A-C) Normalization of the pHs of cancer-cell-cultured medium to 7.4 results in decreased CAF activation. Human MSCs were cultured with original or normalized medium for a week. The " $n$ " before the type of cancer cells represents "normalized". (D) Decrease in extracellular pH induces the expression of CAF-specific genes in MSCs. Human MSCs were stimulated with respective culture medium ( $\mathrm{pH}$ as indicated) for a week before qPCR detection. (E) Time-dependent effect of decrease in extracellular $\mathrm{pH}$ for $\mathrm{CAF}$ activation is shown. Human MSCs were treated with culture medium at $\mathrm{pH}=7.0$ for different time as indicated. (F) Dose-dependent and time-dependent effects of decrease in extracellular $\mathrm{pH}$ for CAF activation is illustrated by immunoblotting. All experiments were performed at least 3 times (every time in triplicate). Error bars represent the SEM. Statistical analysis was performed using one-way ANOVA followed by Student's $t$ test. *P $<0.05$ (A) versus SKOV-3. (B) versus MKN1. (C) versus MDA-MB-453. (D) versus $\mathrm{pH}=7.4$. (E) versus 0 day. 
A
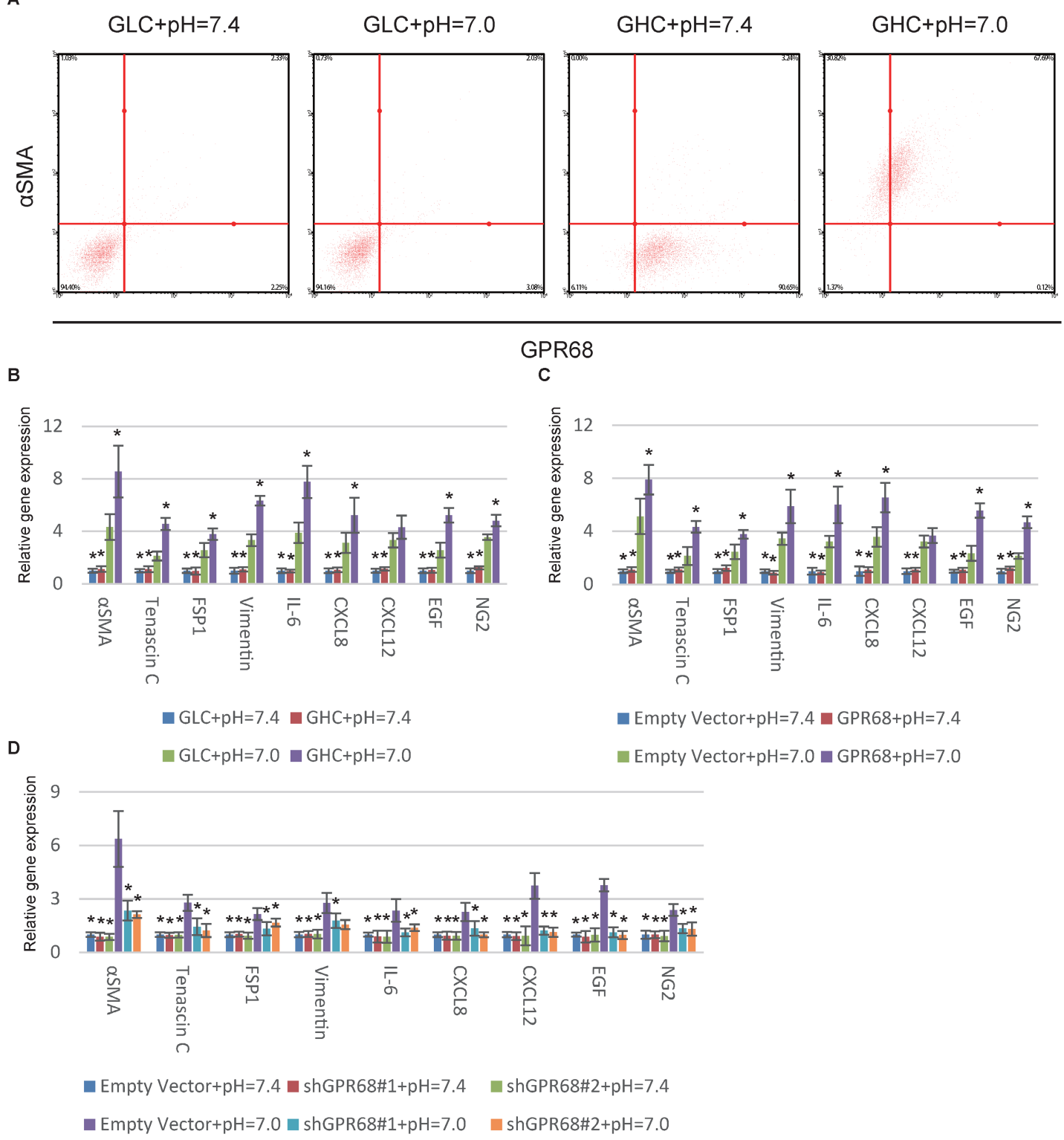

GPR68

C

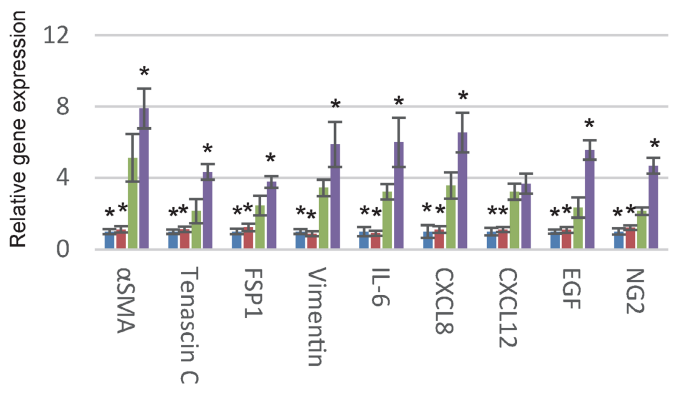

Empty Vector+pH=7.4 a GPR68+pH=7.4

Empty Vector $+\mathrm{pH}=7.0$ GPR68+pH=7.0

Figure 2. GPR68 in regulation of CAF activation. (A) Decrease in extracellular pH promotes CAF activation of GPR68-high clones (GHCs) instead of GPR68-low clones (GLCs). Different clones of MSCs with differential expression of GPR68 were cultured at $\mathrm{pH}=7.0$ for a week. Flow cytometry analysis of $\alpha \mathrm{SMA}$ expression was then conducted. (B) CAF-specific gene expression profiles of GHC and GLC were assessed by qPCR at indicated pHs. (C) Overexpression of GPR68 increased CAF activation at decreased ECF pH. The expression of CAF-specific genes in human MSCs infected with empty or GPR68 vectors were assessed by qPCR. (D) Knockdown of GPR68 blocks pH-dependent regulation on CAF activation. Human MSCs infected with control shRNA or GPR68 shRNA were cultured at indicated pH. All experiments were performed at least 3 times (every time in triplicate). Error bars represent the SEM. Statistical analysis was performed using one-way ANOVA followed by Student's t test. *P < 0.05 (B) versus GLC+pH=7.0. (C, D) versus Empty Vector $+\mathrm{pH}=7.0$.

GPR68 is required for pH-dependent CAF activation

Proton-sensing GPCRs, including GPR4 [15], GPR65 [16], GPR68 [17] and GPR132 [18] are commonly activated under pHs from 6 to 8 through histidine residues [19]. GPR68 is the solely known proton-sensing GPCRs expressed by MSCs [14]. We therefore hypothesized that GPR68 might mediate the $\mathrm{CAF}$ activation in response to decreased $\mathrm{pH}$. We stimulated different MSC clones with differential expression of GPR68. The MSCs from clones with high
GPR68 expression can transform into CAFs under decreased ECF pH. In contrast, MSCs with low GPR68 expression were insensitive to $\mathrm{pH}$ change (Fig. 2A). Detection of CAF-specific genes further supported this observation (Fig. 2B). We then manipulated the expression of GPR68 by lentivirus infection in MSCs. Overexpression of GPR68 elicited increased sensitivity to $\mathrm{pH}$ change in MSCs (Fig. 2C and S2A). Consistently, silence of GPR68 blocked CAF activation under decreased ECF pH (Fig. 2D and S2B). 


\section{GPR68 promotes MSC-CAF transition via YAP activation}

YAP is a tension-sensed CAF activator that promotes malignancy through a mechanically reinforced feed-forward loop [20]. We previously reported that GPR68 could activate YAP under decreased ECF $\mathrm{pH}$ through Rho signalling in MSCs [14]. Hence we hypothesized YAP might be also vital in proton-sensed mechanism of CAF activation. Supporting our notion, interfering with Rho signalling with botulinum toxin C3 and Y27632, specific inhibitors of Rho GTPases and ROCK, blocked pH-dependent CAF activation (Fig. 3A). We then conducted YAP overexpression in MSCs and observed a significant upregulation of CAF-specific genes (Fig. 3B and S3A). Silence of YAP resulted in decreased expression of CAF-specific genes under both normal and acidic $\mathrm{pHs}$ (Fig. 3C and S3B). Taken together, we concluded that decreased ECF $\mathrm{pH}$ could promote CAF activation of MSCs through GPR68-YAP signalling.

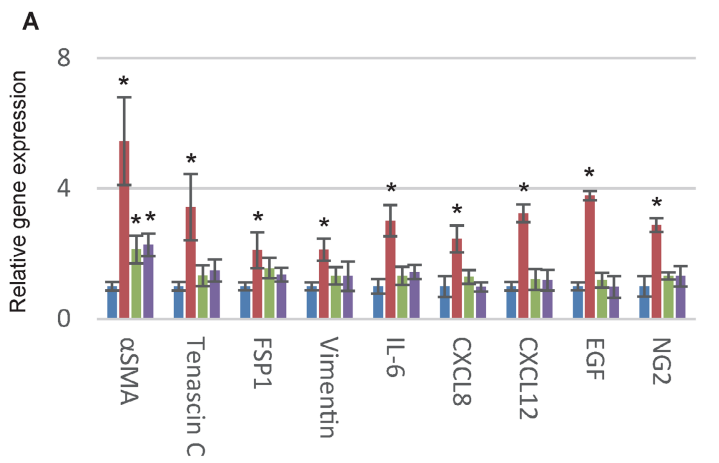

$\mathrm{pH}=7.4 \square \mathrm{pH}=7.0 \square \mathrm{pH}=7.0+\mathrm{Y} 27632 \square \mathrm{pH}=7.0+\mathrm{C} 3$

$$
\text { C }
$$

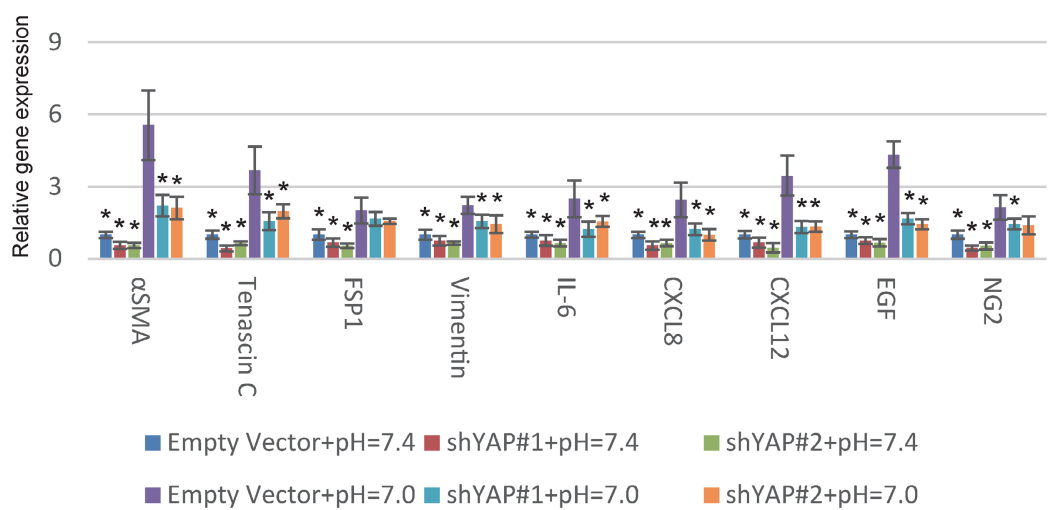

Figure 3. YAP is required for $\mathrm{pH}$-dependent $\mathrm{CAF}$ activation. (A) Inhibition of Rho signalling interferes with $\mathrm{CAF}$ activation in response to decreased extracellular $\mathrm{pH}$. Human MSCs were cultured at indicated $\mathrm{pHs}$ and treated with $\mathrm{C} 3(3 \mu \mathrm{g} / \mathrm{mL})$ or $\mathrm{Y} 27632(50 \mathrm{mM})$ in medium for one week. (B) Overexpression of YAP with lentiviruses increases the expression of CAF-specific genes. (C) Silence of YAP blocks CAF activation in response to decreased pH. All experiments were performed at least 3 times (every time in triplicate). Error bars represent the SEM. Statistical analysis was performed using one-way ANOVA followed by Student's $t$ test. *P $<0.05$ (A) versus $\mathrm{pH}=7.4$. (B) versus Empty Vector. (C) versus Empty Vector $+\mathrm{pH}=7.0$.

\section{Knockdown of GPR68 suppresses CAF activa- tion in cancer microenvironment}

Next, we investigated whether interference of GPR68 can decrease CAF activation under cancer microenvironment. We therefore co-cultured SKOV-3 cells with GPR68-silenced MSCs to determine whether GPR68 knockdown inhibited CAF activation in cancer microenvironment. The proportion of highly aSMA-expressed MSCs was significantly decreased after GPR68 interference (Fig. 4A). We co-transplanted MDA-MB-453 cells and MSCs in nude mice for tumour formation. The CAF activation was inhibited after GPR68 knockdown determined by flow cytometry analysis of aSMA (Fig. 4B). We then sorted out GFP-positive cells by flow cytometry from transplanted tumour for further analyses on phenotypes. GPR68-silenced MSCs expressed less CAF-specific markers and tumour-promoting factors compared with empty vector control (Fig. 4C). In addition, the secretion of tumour-suppressing factors from MSCs was preserved after GPR68 knockdown.

B

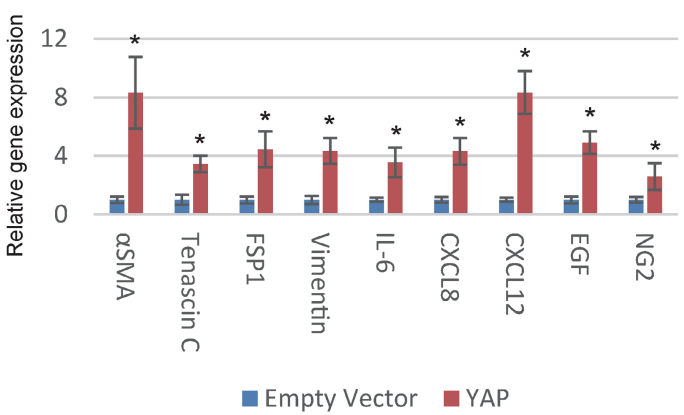


A

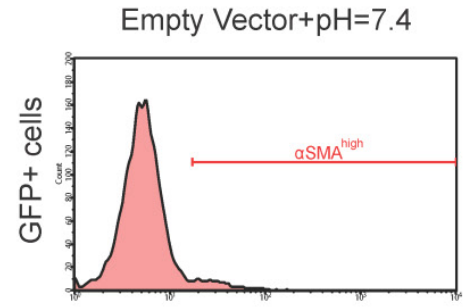

Empty Vector $+\mathrm{pH}=7.0$

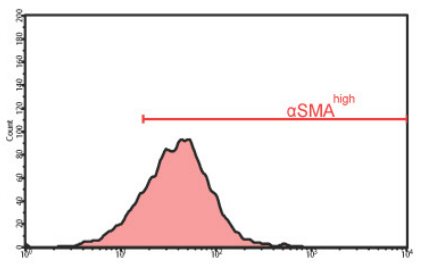

shGPR68

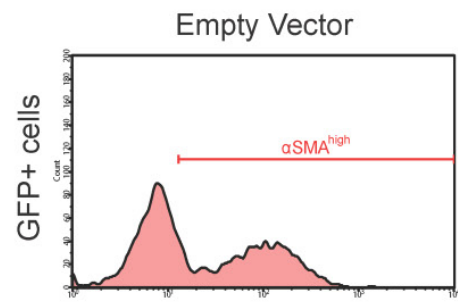

C

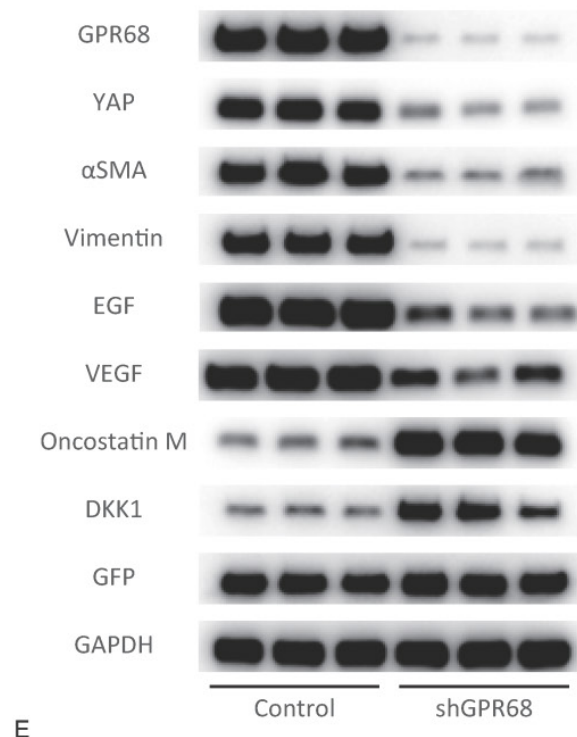

E

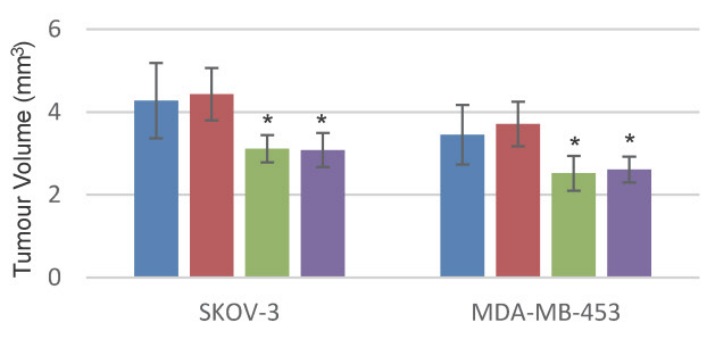

- Control = Empty Vector = shGPR68\#1 = shGPR68\#2
D

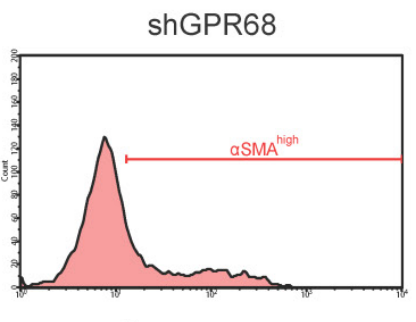

B

F
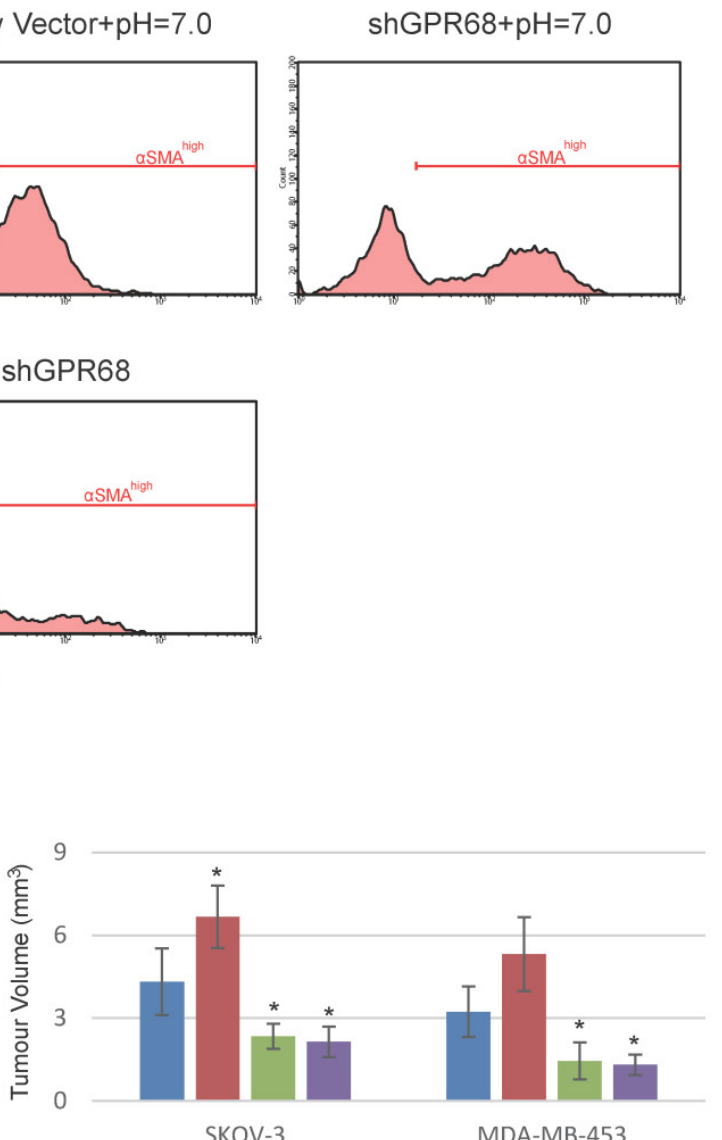

- Control a Empty Vector = shGPR68\#1 = shGPR68\#2

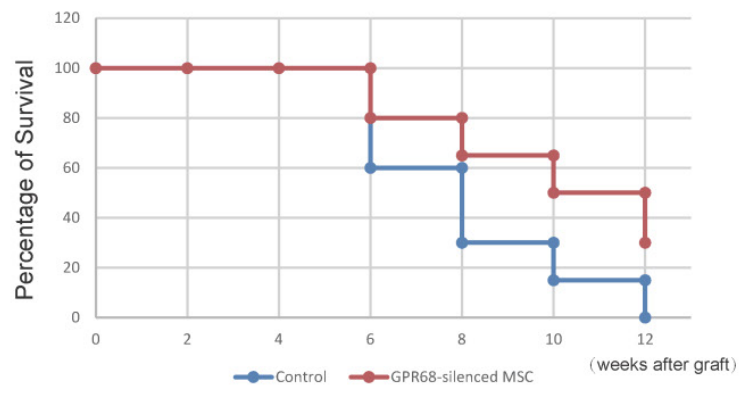

Figure 4. Silence of GPR68 in MSCs suppresses tumour growth. (A) CAF activation of MSCs co-cultured with SKOV-3 cells was inhibited after the silence of GPR68. GFP-labelled MSCs were co-cultured with SKOV-3 cells for one week before flow cytometry detection. (B) CAF activation of GPR68-silenced MSCs in breast carcinoma was suppressed. GFP-labelled MSCs with or without GPR68 silencing were injected with MDA-MB-453 cells subcutaneously in nude mice ( $n=10)$. The expression of aSMA in GFP-labelled MSCs was detected by flow cytometry after sacrifice. (C) GFP-positive cells were sorted out from each tumour by flow cytometry and Western blot was then conducted for phenotype analysis. (D) Tumour volume was also assessed and significantly decreased in GPR68-silenced groups. (E) Venous injection of GPR68-silenced MSCs inhibited tumour growth in situ $(n=10)$. Error bars represent the SEM. (F) The mice received GPR68-silenced MSCs showed prolonged survival time after the graft of SKOV-3. Statistical analysis was performed using log-rank test or one-way ANOVA followed by Student's t test. *P $<0.05$ versus control.

\section{GPR68-silenced MSCs as a therapy for cancer}

Due to the increased secretion of tumour-suppressing factors, the tumour-promoting effects of MSC co-transplantation was reversed after
GPR68 silencing (Fig. 4D). The inflammatory tumour microenvironment enables circulating MSCs to specifically home to tumour tissues while avoiding peritumoural normal tissues [21]. We therefore assumed 
that intravenous injection of GPR68-silenced MSCs might be a potential therapy for cancer. We then assessed whether systemic transplantation of GPR68-silenced MSCs could suppress tumour progression. Tumour growth in situ was inhibited after systemic transplantation of GPR68-silenced MSCs (Fig. 4D and Fig. S4). In addition, the mice received systemic transplantation of GPR68-silenced MSCs showed prolonged survival time compared with control group (Fig. 4E).

\section{Discussion}

Tumour growth is highly dependent on complicated and dynamic interactions between cancer cells and tumour stroma mediated by direct contact and secreted factors. MSCs as an important stromal component might exhibit extremely differential functions according to different microenvironments. Although naive MSCs supress tumour growth, the MSCs can also be transformed into CAFs by tumour microenvironment and further promotes tumour progression. As a result, highly controversial results have been reported in previous studies that addressed the role of MSCs in tumour development and progression.

The current understanding on the mechanism of CAF activation is limited to soluble factor and mechanotransduction. Here we reveal the third player which is the acidy extracellular $\mathrm{pH}$. Our study demonstrated that acidic microenvironments caused by lactate production of cancer cells promotes the CAF activation of MSCs. The acidification of the extracellular environment promotes CAF activation of MSCs, which further stimulates cancer cell proliferation. Increased proliferation of cancer cells exacerbates the hypoxic and acidy microenvironment which further promotes CAF activation of MSCs. Our finding provides a novel insight into CAF activation and might illustrate many unexplained problem in $\mathrm{CAF}$ biology after further investigation.

We then investigated the underlying mechanism of $\mathrm{pH}$-dependent CAF activation. Recent discoveries on proton-sensing GPCRs extended proton-sensing mechanisms from neurons to other types of cells. Unlike proton-sensing ionotropic channels primarily expressed by neurons, proton-sensing GPCRs are expressed by many types of non-neural cells. Multiple previous studies have demonstrated that acidic ECF pose significant influences on a wide range of biological behaviours, including proliferation, adhesion [22], and secret production [23-25] through proton-sensing GPCR signalling. We reported decreased extracellular $\mathrm{pH}$ promotes proliferation and survival of MSCs through GPR68-YAP axis in previous study [14]. Here we demonstrated that this axis is also responsible for $\mathrm{pH}$-dependent CAF activation of MSCs. Silence of
GPR68 in MSCs inhibits CAF activation under tumour microenvironment, which break the viscous circle. Untransformed MSCs secrete more tumour-supressing factors including DKK1 and oncostatin $\mathrm{M}$ and finally supress tumour growth.

Previous reports have shown that CAF activation is associated with cancer metastasis [26, 27]. It is conceivable that cancer cell invasion can lead to local hypoxia and acidification which promotes CAF activation of local MSCs. We therefore hypothesize that GPR68-YAP signalling might be also involved in metastasis of cancer cells and currently work on the investigation of this issue.

\section{Supplementary Material}

Figures S1-S4, and Supplementary Table 1. http://www.ijbs.com/v12p0389s1.pdf

\section{Acknowledgement}

We thank Drs. Xiaolin Liu, Shumin Zhou, and Juan Liu for constructive comments; Dr. Junjie Guan for technical help. This work was supported by the Special Fund for Healthy-scientific Research in the Public Interest, China (201402016) and the National Natural Science Foundation of China (81301589).

\section{Authors' contributions}

C.Z. and H.Z. designed the experiments and analysed the data. S.T. and J.Y. isolated the MSCs. S.T. and Y. Z. performed all of the qPCR detections. W.Y. and $\mathrm{Y}$. W. conducted in vivo study. H.Z. conducted the rest of the experiments. C.Z. and H.Z. wrote the manuscript.

\section{Competing interests} interests.

The authors declare that they have no competing

\section{References}

1. Chang HY, Sneddon JB, Alizadeh AA, Sood R, West RB, Montgomery K, et al. Gene expression signature of fibroblast serum response predicts human cancer progression: similarities between tumors and wounds. PLoS biology. 2004; 2: E7.

2. Cirri P, Chiarugi P. Cancer associated fibroblasts: the dark side of the coin. American journal of cancer research. 2011; 1: 482-97.

3. Spaeth EL, Dembinski JL, Sasser AK, Watson K, Klopp A, Hall B, et al. Mesenchymal stem cell transition to tumor-associated fibroblasts contributes to fibrovascular network expansion and tumor progression. PloS one. 2009; 4: e4992.

4. Sugimoto $\mathrm{H}$, Mundel TM, Kieran MW, Kalluri R. Identification of fibroblast heterogeneity in the tumor microenvironment. Cancer biology \& therapy. 2006; 5: 1640-6.

5. Augsten M. Cancer-associated fibroblasts as another polarized cell type of the tumor microenvironment. Frontiers in oncology. 2014; 4: 62.

6. Ishii G, Ochiai A, Neri S. Phenotypic and functional heterogeneity of cancer-associated fibroblast within the tumor microenvironment. Advanced drug delivery reviews. 2015

7. Orimo A, Gupta PB, Sgroi DC, Arenzana-Seisdedos F, Delaunay T, Naeem R, et al. Stromal fibroblasts present in invasive human breast carcinomas promote tumor growth and angiogenesis through elevated SDF-1/CXCL12 secretion. Cell. 2005; 121: 335-48.

8. Wang ML, Pan CM, Chiou SH, Chen WH, Chang HY, Lee OK, et al. Oncostatin $\mathrm{m}$ modulates the mesenchymal-epithelial transition of lung adeno- 
carcinoma cells by a mesenchymal stem cell-mediated paracrine effect. Cancer research. 2012; 72: 6051-64.

9. Zhu Y, Sun Z, Han Q, Liao L, Wang J, Bian C, et al. Human mesenchymal stem cells inhibit cancer cell proliferation by secreting DKK-1. Leukemia. 2009; 23: 925-33.

10. Weber CE, Kothari AN, Wai PY, Li NY, Driver J, Zapf MA, et al. Osteopontin mediates an MZF1-TGF-beta1-dependent transformation of mesenchymal stem cells into cancer-associated fibroblasts in breast cancer. Oncogene. 2014.

11. Mishra PJ, Mishra PJ, Humeniuk R, Medina DJ, Alexe G, Mesirov JP, et al. Carcinoma-associated fibroblast-like differentiation of human mesenchymal stem cells. Cancer research. 2008; 68: 4331-9.

12. Talele NP, Fradette J, Davies JE, Kapus A, Hinz B. Expression of alpha-Smooth Muscle Actin Determines the Fate of Mesenchymal Stromal Cells. Stem cell reports. 2015; 4: 1016-30.

13. Calon A, Tauriello DV, Batlle E. TGF-beta in CAF-mediated tumor growth and metastasis. Seminars in cancer biology. 2014; 25: 15-22.

14. Zhu H, Cheng X, Niu X, Zhang Y, Guan J, Liu X, Tao S, Wang Y, Zhang C. Proton-sensing GPCR-YAP Signalling Promotes Cell Proliferation and Survival. Int J Biol Sci 2015; 11(10):1181-1189. doi:10.7150/ijbs.12500.

15. Wyder L, Suply T, Ricoux B, Billy E, Schnell C, Baumgarten BU, et al. Reduced pathological angiogenesis and tumor growth in mice lacking GPR4, a proton sensing receptor. Angiogenesis. 2011; 14: 533-44.

16. Li Z, Dong L, Dean E, Yang LV. Acidosis decreases c-Myc oncogene expression in human lymphoma cells: a role for the proton-sensing $G$ protein-coupled receptor TDAG8. International journal of molecular sciences. 2013; 14: 20236-55.

17. Mogi C, Nakakura T, Okajima F. Role of extracellular proton-sensing OGR1 in regulation of insulin secretion and pancreatic beta-cell functions. Endocrine journal. 2014; 61: 101-10.

18. Murakami N, Yokomizo T, Okuno T, Shimizu T. G2A is a proton-sensing G-protein-coupled receptor antagonized by lysophosphatidylcholine. The Journal of biological chemistry. 2004; 279: 42484-91.

19. Ludwig MG, Vanek M, Guerini D, Gasser JA, Jones CE, Junker U, et al. Proton-sensing G-protein-coupled receptors. Nature. 2003; 425: 93-8.

20. Maller O, DuFort CC, Weaver VM. YAP forces fibroblasts to feel the tension. Nature cell biology. 2013; 15: 570-2.

21. Luo J, Ok Lee S, Liang L, Huang CK, Li L, Wen S, et al. Infiltrating bone marrow mesenchymal stem cells increase prostate cancer stem cell population and metastatic ability via secreting cytokines to suppress androgen receptor signaling. Oncogene. 2014; 33: 2768-78.

22. Chen A, Dong L, Leffler NR, Asch AS, Witte ON, Yang LV. Activation of GPR4 by acidosis increases endothelial cell adhesion through the cAMP/Epac pathway. PloS one. 2011; 6: e27586.

23. Mogi C, Tobo M, Tomura H, Murata N, He XD, Sato K, et al. Involvement of proton-sensing TDAG8 in extracellular acidification-induced inhibition of proinflammatory cytokine production in peritoneal macrophages. Journal of immunology. 2009; 182: 3243-51.

24. He XD, Tobo M, Mogi C, Nakakura T, Komachi M, Murata N, et al. Involvement of proton-sensing receptor TDAG8 in the anti-inflammatory actions of dexamethasone in peritoneal macrophages. Biochemical and biophysical research communications. 2011; 415: 627-31

25. Dong L, Li Z, Leffler NR, Asch AS, Chi JT, Yang LV. Acidosis activation of the proton-sensing GPR4 receptor stimulates vascular endothelial cell inflammatory responses revealed by transcriptome analysis. PloS one. 2013; 8: e61991.

26. Kuchnio A, Moens S, Bruning U, Kuchnio K, Cruys B, Thienpont B, et al. The Cancer Cell Oxygen Sensor PHD2 Promotes Metastasis via Activation of Cancer-Associated Fibroblasts. Cell reports. 2015; 12: 992-1005.

27. Zang C, Eucker J, Habbel P, Neumann C, Schulz CO, Bangemann N, et al. Targeting multiple tyrosine kinase receptors with Dovitinib blocks invasion and the interaction between tumor cells and cancer-associated fibroblasts in breast cancer. Cell cycle. 2015; 14: 1291-9. 